Recepción: 09 / 08 / 2018

Aceptación: 17 / 09 / 2018

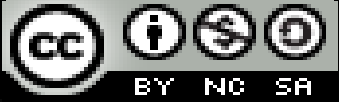

Ciencias económicas y empresariales

Publicación: 01 / 11 / 2018

Artículo de investigación

\title{
El marketing en instituciones del tercer sector
}

\author{
Marketing in third sector institutions \\ Marketing para instituições do terceiro setor
}

Ángela A. Cevallos-Ponce ${ }^{\mathrm{I}}$

angiecevallosp@yahoo.com

Kelly J. Estrada-Realpe II kelly.estrada@utelvt.edu.ec

Correspondencia: angiecevallos@yahoo.com

I Magíster en Administración de Empresas, Ingeniero Comercial, Docente de la Universidad Laica Eloy Alfaro de Manabí, Manta, Ecuador.

${ }^{\text {II } M a g i ́ s t e r ~ e n ~ D o c e n c i a ~ y ~ G e r e n c i a ~ e n ~ E d u c a c i o ́ n ~ S u p e r i o r, ~ D i p l o m a ~ S u p e r i o r ~ e n ~ G e r e n c i a ~ E s t r a t e ́ g i c a ~ d e ~}$ Mercadeo, Licenciada en Publicidad y Mercadotecnia, Docente de la Universidad Luis Vargas Torres de Esmeraldas, Esmeraldas, Ecuador. 


\title{
Resumen
}

El presente artículo tiene como objetivo principal, revisar los conceptos fundamentales del marketing en las instituciones del tercer sector, que son aquellas que tienen una finalidad netamente social, sin fines de lucro. Este trabajo busca cimentar un enfoque acorde con las relaciones de las organizaciones con la sociedad y sus distintos grupos, actividades y público de las organizaciones sociales, procurando distanciar del mercadeo social y evitando la utilización de herramientas y procesos del marketing comercial. Para concluir un lenguaje para esta tipología mercadológica, se realiza un análisis de la literatura referente a las ciencias sociales y políticas, conformando así un contenido sobre asuntos sociales y marketing.

Palabras clave: Marketing; tercer sector; organizaciones sociales; proceso de selección; índice de rotación; cooperativa; acciones correctivas.

\begin{abstract}
The main objective of this article is to review the fundamental concepts of marketing in thirdsector institutions, which are those that have a purely social, non-profit purpose. This work seeks to cement an approach consistent with the relationships of organizations with society and its different groups, activities and public of social organizations, trying to distance themselves from social marketing and avoiding the use of commercial marketing tools and processes. To conclude a language for this marketing typology, an analysis of the literature referring to the social and political sciences is carried out, thus forming a content on social issues and marketing.
\end{abstract}

Keywords: Marketing; third sector; Social Organizations; selection process; rotation index; cooperative; corrective actions.

\section{Resumo}

O objetivo principal deste artigo é revisar os conceitos fundamentais de marketing nas instituições do terceiro setor, que são aquelas que têm uma finalidade puramente social e sem fins lucrativos. Este trabalho busca cimentar uma abordagem consistente com as relações das organizações com a sociedade e seus diferentes grupos, atividades e públicos de organizações sociais, procurando se distanciar do marketing social e evitando o uso de ferramentas e processos de marketing comercial. Para concluir uma linguagem para essa tipologia de marketing, é 
realizada uma análise da literatura referente às ciências sociais e políticas, formando um conteúdo sobre questões sociais e de marketing.

Palavras chave: Marketing; terceiro setor; organizações sociais; processo de seleção; índice de rotação; cooperativa ações corretivas.

\section{Introducción}

Las perspectivas de las empresas que conforman el tercer sector, conocidas como Entidades No Lucrativas, ENL, son bastante negativas. Sus principales suministradores de recursos financieros: las Administraciones Públicas, las empresas, sus socios y sus benefactores individuales, se encuentran atravesando una profunda crisis caracterizada por la palabra "recorte".

Debido al mismo panorama social, el mercado de las instituciones del tercer sector aumenta, puesto que crece la cantidad de necesidades que atender, mostrando a su vez, tipología y características diferentes.

Con lo mencionado, se entiende que la mayoría de las ONG, de las Entidades No Lucrativas, perciban la exigencia de transformar sus estrategias para convertirse en organizaciones que prestan servicios, encajando en el concepto más comercial del término, siendo en esta evolución donde el Marketing debe adquirir la importancia que deberá tener en el nuevo escenario de mercado.

En la actualidad, el marketing es un concepto muy conocido y estudiado en muchos centros educativos, pero su marco teórico se ha basado sobre todo en el ámbito comercial y en sus distintas herramientas para conquistar mercados y posicionar marcas, desconociendo sus posibilidades en ámbitos distintos al comercial. En Latinoamérica, la falta de conocimiento en materias organizacionales y metodológicas, y la carente investigación científica en sectores como el social, político, electoral, etc., han reducido los beneficios a los fines sociales, mientras que en otros entornos, estos sectores ya explotan las bondades y ventajas que el marketing ofrece. Por otra parte, existen empresas con fines de lucro que hacen uso de un expansivo marketing supuestamente social o con ideales sociales, al tiempo que sus ventas y utilidades reflejan un incremento. 
Las universidades son las que tienen la misión de formar a profesionales que lleven a las organizaciones sociales los beneficios del marketing en instituciones del tercer sector. Un buen comienzo es obligatoriedad de la práctica social en algunas instituciones de educación superior y el refuerzo a sus conocimientos mediante los seminarios sociales.

El concepto de que una aproximación al marketing en ámbitos no lucrativos exige un claro conocimiento institucional (sociedad civil organizada, Estado, mercado y sociedad), una comprensión de la realidad social en la que se enmarcan las instituciones mencionadas y un reconocimiento de las responsabilidades de sus organizaciones en el propósito de garantizar mejores niveles en la calidad social.

Para un mejor entendimiento, es necesario identificar no solo los distintos grupos que conforman sistema de valor del tercer sector, sino también de las contribuciones realizadas por cada uno, conocimiento acerca del mercado social, las variables que enfocan al marketing de las instituciones sociales, y las diferencias entre el marketing de empresas y de organizaciones sociales, y se destaca la pertinencia de la apropiación de conceptos y su correspondiente contextualización a las especificidades del tercer sector, donde operan organizaciones sociales como fundaciones, centros de ayuda, hogares infantiles, albergues, etc.

Por tanto, se pretende enmarcar propios enfoques de las ciencias sociales y políticas, buscando aportar términos adecuados y pertinentes para el marketing de instituciones sociales. Se requiere un análisis de la literatura especializada existente (institucionalismo, política social, bienes públicos, pobreza y vulnerabilidad) y su contraste con la realidad del tercer sector y de las organizaciones sociales.

\section{Desarrollo}

Una serie de amenazas y de oportunidades se ciernen sobre las instituciones que conforman el tercer sector, lo que les va a obligar a acometer profundas transformaciones, tanto en la propia filosofía que las rige, como en las actuaciones internas y externas que realicen. (Barranco J., 2008)

Al conjunto de Entidades No Lucrativas, (ENL, ONG, Fundaciones, etc.), que actúan en el Mercado Social se le denomina Tercer Sector para diferenciarlo de los sectores público y privado. 
Es una acepción que se debe a W.A. Nielsen y que se generalizó, a partir de 1.975, como consecuencia de su "oficialización" por parte de la Comisión Filler.

El Estado, el mercado y la sociedad civil han sido reconocidas como instituciones complementarias para lograr el desarrollo social de las naciones. Esto implica que los Gobiernos comparten la responsabilidad de proporcionar bienestar con distintos actores como agencias comunitarias, empresas y entidades sociales. En este sentido, ya no solo los Gobiernos, sino también las organizaciones de la sociedad civil juegan un papel importante en la entrega de bienes, productos y servicios de carácter social (Toca C., 2013). Convertir el bienestar social en un asunto de todos (agentes, actores e individuos), se reconoce como un proceso de socialización del bienestar. (Wong y Tang, 2006/2007)

El tercer sector está enmarcado en la sociedad civil, donde la tendencia indica que la organización debe trabajar coordinadamente con los grupos de apoyo para cumplir su misión, pues son ellos los que proporcionan los medios y recursos necesarios para implementar sus programas, políticas y proyectos sociales que beneficiarán a las poblaciones vulnerables. No existe solo una relación directa entre los grupos de apoyo y la organización social y entre esta y los beneficiarios, sino también una relación indirecta entre los grupos de apoyo y los beneficiarios. (Toca C., 2013)

\section{Relaciones de las organizaciones con la sociedad y sus distintos grupos}

Varios enfoques han sido dispuestos para estudiar y administrar las relaciones de las organizaciones con la sociedad y sus distintos grupos, destacando la perspectiva de los stakeholders y el marketing. Ambos se orientan a resaltar problemas, establecer respuestas y mantener un equilibrio entre las necesidades de estos grupos y el cumplimiento de finalidades sociales. Desde un referente mercadológico, "la creación de valor está determinada por una relación equilibrada entre contraprestaciones (aquello que ofrece la organización a los stakeholders) y contribuciones (lo proporcionado por los stakeholders a la organización)". (Lorca, 2004, p. 52).

Las relaciones con stakeholders son de total relevancia para el éxito de la organización, incluyen más que intereses, se extienden a valores y reclamos, así como a la habilidad y poder para influir. Los grupos de apoyo exigen un tratamiento especial, pues debido a que no reciben una 
contraprestación monetaria por facilitar ciertos recursos (como intereses o rendimientos), no se les puede exigir la disponibilidad de estos por más que las necesidades sociales sean apremiantes. En términos sencillos, como la retribución de los fondos no es exigible, tampoco lo será su disposición. (Martín y Martínez, 2004)

Puesto que son los socios y los fundadores quienes representan a estas instituciones, serán los responsables de afianzar y fortalecer las relaciones con los stakeholders internos y externos. Esto no implica que deba desconocerse su labor, cuando de sistema de valor se trata, ya que sus recursos (dinero, tiempo y materiales) constituyen la principal fuente para el desarrollo de las actividades de la entidad. De manera general, estos socios y fundadores forman parte del principal órgano de dirección cuya denominación puede ser la de junta directiva, junta de socios o patronato. Sus aportes periódicos constituyen una fuente importante para la sostenibilidad de la entidad, además de garantizar la disponibilidad de cierta cantidad de recursos. Normalmente, estos fondos son requeridos para enfrentar gastos de administración y de funcionamiento, de modo que las donaciones y transferencias van directamente a los beneficiarios garantizando así la debida focalización de los recursos. Sin embargo, es posible que el trabajo de una organización social no se logre cubrir en su totalidad con el voluntariado, por lo que en ocasiones es necesario contratar empleados para el desempeño de ciertas actividades operativas o profesionales (personal remunerado). (Toca C., 2013)

Uno de los problemas que enfrentan las entidades del tercer sector es la alta rotación de personal, que dificulta la continuidad de los programas" (Martín y Martínez, 2004, p. 81), lo que puede convertirse en un aspecto negativo para los grupos de apoyo, repercutiendo en la credibilidad y eficiencia de los costos.

El hecho que la organización tenga un fin social, no exime a estas organizaciones del cumplimiento de sus responsabilidades patronales, es decir, pagar salarios justos con jornadas laborales normales, incorporar programas de incentivos y contar con políticas laborales humanas. Estas deben convertirse en una opción atractiva para el desempeño de los profesionales.

El voluntariado es la asignación de tiempo para desempeñar actividades operativas, técnicas o profesionales en la organización, sin recibir remuneración y sin mediar exigencias. Los voluntarios se pueden dedicar a distintas actividades: cuidado y atención de los beneficiarios, 
capacitación en ciertos oficios y labores o prestación de un servicio profesional. Los voluntarios han sido categorizados de acuerdo con las funciones desempeñadas en los siguientes grupos:

- Los estudiantes o pasantes, en el marco de la práctica social, quienes desempeñan tareas administrativas como contaduría, administración, y comunicación

- Los recaudadores de fondos (fundraisers) dedicados a la consecución de fondos y recursos con destino a los programas y proyectos de causa social

- Los profesionales dedicados a la atención directa de la población beneficiaria (médicos, psicólogos, terapeutas, trabajadores sociales, etc.)

- Los relacionistas, que contribuyen en labores logísticas y operativas para la realización de los distintos eventos institucionales (cenas, subastas, etc.).

Partiendo de la idea de que las donaciones son entregas, traspasos o cesiones voluntarias y con destino a la población necesitada, estas pueden ser confiadas por individuos, grupos familiares y empresas a una organización social para su respectiva canalización (donantes individuales y empresariales). Estas pueden ser transferencias de recursos, como dinero; aportaciones con productos y bienes esenciales, generalmente costosos para la atención del beneficiario, como alimentos, ropa, electrodomésticos, artículos para el hogar, juguetes, elementos hospitalarios, etre otros; trabajo, reflejado en la donación de servicios profesionales.

El Gobierno en sus 3 niveles (nacional, provincial y municipal) se configura en un grupo de interés muy importante para las organizaciones sociales. Las subvenciones oficiales, las transferencias gubernamentales y las exenciones constituyen las modalidades de ayuda más conocidas. La limitante de las primeras es que este tipo de ayuda en ocasiones se encuentra etiquetada, es decir, su destino se orienta hacia una causa social, un programa o proyecto social particular (salud, educación y protección de cierto tipo de población vulnerable). Esto explica por qué algunas causas sociales se financian con una buena proporción de fondos oficiales y otras nunca reciben ayuda del Gobierno.

Las agencias de ayuda internacional brindan apoyo a aquellas iniciativas y esfuerzos locales capaces de cambiar la condición de vulnerabilidad de una determinada población. Los fondos y las agencias de ayuda internacional captan y canalizan recursos hacia aquellas entidades 
encargadas de implementar programas y prácticas para atender necesidades urgentes de la población. Dentro de las más importantes destacan: agencias de ayuda oficial para el desarrollo, agencias de las Naciones Unidas, bancos multilaterales de desarrollo, fundaciones internacionales, corporaciones globales, organizaciones no gubernamentales internacionales, movimientos sociales mundiales, organizaciones internacionales religiosas, Fondo Monetario Internacional, Banco Mundial y Organización Mundial del Comercio.

El beneficiario es aquel que resulta favorecido de los bienes, servicios, políticas o programas sociales, aquel que dada su condición de miembro vulnerable de una sociedad percibe una prestación social. La ubicación de los beneficiarios en el seno del denominado sector informal es la que hace que el beneficio proporcionado a un grupo o segmento particular irradie a toda la sociedad. Implica, entonces, que los favorecidos no son solamente los beneficiarios, sino todos los miembros de la sociedad. Los beneficiarios se convierten en un referente muy importante cuando se trata de evaluar el impacto social de una organización, por lo que no se descarta que su selección se realice a partir de estudios socioeconómicos y geográficos y con el diseño de mapas de pobreza que para el caso de programas de alimentación identifica poblaciones en estado de desnutrición y marginalidad. (Barquera, Rivera y Gasca, 2001)

La participación de los beneficiarios, que se suma a la de empleados y voluntarios, y que ha sido llamada la construcción conjunta de la oferta y la demanda, es característica de toda organización social. Esta participación tiene un carácter político de definición de las necesidades en la esfera pública y apoya en la construcción de capital social en las comunidades (Jean-Louis Laville, 1994, citado en Richer y López, 2004). La participación de los beneficiarios de los programas se identifica como un elemento esencial para la implementación de los mismos, y en lo posible debe trascender a los procesos de planeación, control y evaluación (Barquera et al., 2001).

\section{Producto social}

Uno de los aspectos más importantes a los que se enfrenta una entidad del Tercer Sector es el referente al Producto o Servicio Social que puede ofrecer a sus públicos objetivos, a sus clientes.

Desde la perspectiva de Marketing, esto es lo que le va a permitir diferenciarse de sus competidores, ser conocido por la Sociedad, establecer la adecuada estrategia de Comunicación 
y, como consecuencia de todo ello, posicionarse positivamente en un mercado tan proceloso como es el de la solidaridad. (Barranco J., 2012)

Es importante identificar que el Producto Social es distinto en función de los grupos de interés de la entidad: beneficiarios o benefactores, además de otros según las características de la misma. De ahí la importancia de una buena Política de Producto Social en este tipo de instituciones.

El producto social debe constituir, en esencia, la oferta que aporte el valor que espera la demanda o, dicho de otro modo, la adaptación de esta oferta para que satisfaga las necesidades que tenga el mercado.

La actividad de la ENL va a girar alrededor de un producto social, tangible o intangible, que es el que va a ofrecer a su mercado.

Como objetivo fundamental, esta política debe brindar a sus clientes un producto atractivo, específico, que le ayude a diferenciarse positivamente y que permita ser utilizado en el diseño y desarrollo de una adecuada estrategia de Comunicación.

Es importante establecer el equilibrio entre la oferta y demanda, ya que de otra manera la ENL llegará a ser una institución bienintencionada pero sin viabilidad futura.

En la mayoría de las organizaciones del Tercer Sector, el producto no es necesariamente un bien tangible, sino una conjunción de un ideal solidario y de un producto o servicio que llega físicamente a los clientes. Si no se es conocedor de la relevancia de combinar ambos elementos, se llegaría a perder la dimensión de lo que supone la acción solidaria.

La gestión de un producto social propio es la base de la construcción de la identidad de la ENL, lo que permitirá que, ante cualquier referencia a un producto similar surja, espontáneamente, la identificación privilegiada con la entidad.

\section{Organización}

Para establecer que la estructura organizativa de la ENL tenga una visión de Marketing, es necesario que todos quienes la conforman tengan claro que su actividad debe estar orientada a satisfacer las expectativas de sus públicos objetivos, siendo imprescindible que la entidad y su organización se especialicen en segmentos concretos de su mercado social. De esta condición se 
deriva el apremio, cada vez mayor, de profesionalizar tanto sus efectivos personales como sus métodos de gestión, aprovechando las oportunidades que, para ello, ofrecen las TICs.

Esto va a requerir organizaciones flexibles y altos niveles de participación que generen integración y motivación; lo que implicará, desde la perspectiva de los RRHH, la aplicación estricta de los principios del Marketing Interno. (Barranco J., 2012)

El que las funciones básicas de la entidad se limiten a prestar un servicio social y a captar fondos para poder hacerlo, o que tenga objetivos más intangibles como la modifícación de comportamientos sociales, o que sean las propias de una Fundación corporativa o social, va a implicar un tipo diferente de cliente, una actuación respecto a él distinta y unos canales estructurados de forma que faciliten el acceso a los clientes de la institución.

\section{Variables del marketing en el tercer sector}

El marketing de organizaciones sociales se define como el conjunto de herramientas o variables controlables que, se identifica en 4 variables: oferta social, contribución, asignación y comunicación.

\section{Oferta social}

El tercer sector ofrece a la sociedad una serie de servicios intangibles que permiten dar cumplimiento a su finalidad original o complementaria. Esta oferta puede materializarse a través de bienes públicos, preferentes o meritorios, de políticas públicas o de políticas y programas sociales. Un bien público es un beneficio proporcionado a la comunidad o colectividad no solo por las entidades del Gobierno, sino también por las organizaciones sociales. Cuando los programas de vacunación de los menores son impuestos por el Estado y cuando la sociedad considera que el cuidado de la salud de los menores cuyos padres no están en condición de adquirirlos es lo más conveniente, la promoción de la salud de los menores se configura como un bien público. Los bienes preferentes y meritorios se ofrecen bajo la forma de servicios, ideas (creencias, actitudes, valores) y prácticas (posturas, comportamientos, conductas). Estas últimas (ideas y prácticas) podrían asimilarse a los desarrollos que en su momento realizaron Kotler y Roberto (1992), pero teniendo claro que no fueron propuestas en el marco de los bienes preferentes y meritorios (públicos). 


\section{Contribución}

Este concepto se enmarca en una denominación de intercambio, definido como el proceso mediante el cual una parte entrega algo de valor a otra y recibe a cambio algo equivalente. Aplicando la definición al contexto social, implicaría que los beneficiarios, como receptores de la oferta social, deben efectuar un aporte que puede ser monetario o no monetario. Se recomienda cuotas de recuperación o cobros mínimos de dinero a los beneficiarios, para garantizar el buen uso de la oferta y eliminar la perpetuidad en la condición de beneficiarios.

Los recursos con destino a los programas sociales deberán originarse de alguna fuente, frente a la dificultad de proporcionarlos en su totalidad por la entidad social. Es entonces cuando los aportes de los grupos de apoyo cobran especial relevancia para financiar los bienes, políticas o programas sociales. Hay que tener claro la diferencia entre contribuciones y aportes, aclarando que las primeras son aportadas por los beneficiarios de la oferta social y los segundos son proporcionados por un grupo que apoya la causa u organización social.

\section{Asignación}

El traspaso de los beneficios provenientes de los aportes de los grupos de apoyo se realiza a través de las políticas y los programas que las entidades sociales instauran para los grupos de beneficiarios. El suministro de beneficios es indirecto para grupos de apoyo y directo para entidades sociales, siendo que estas últimas, por lo general, se apoyan en otros agentes cuando son diversos los servicios ofrecidos a los beneficiarios. En ocasiones, estos agentes forman parte de la organización social, en otras, actúan como intermediarios de la oferta; por ejemplo, un centro de rehabilitación social, puede contar con centros médicos, alojamiento, ayuda psicológica, alimentación, entre otros servicios para atender al beneficiario. En su deseo por comprometerse con determinadas causas sociales (responsabilidad social, relaciones públicas, filantropía o como la quieran denominar las empresas), colegios, centros médicos, centros de formación y capacitación, de carácter privado o particular, pueden prestar servicios a los beneficiarios de la organización social sin cobro pero sí en calidad de aporte. Un ejemplo sería la beca que un colegio privado pone a disposición de un albergue para niños campesinos desplazados. 


\section{Comunicación}

La realidad de los resultados que se obtienen con las Estrategias de Comunicación desarrolladas por una gran parte de las entidades integrantes del Tercer Sector es muy decepcionante.

De los estudios sociológicos realizados se desprenden dos circunstancias bastante generalizables a la gran mayoría de las instituciones que conforman el mismo:

- El público no identifica, claramente, cual es la realidad social de la mayoría de las ONG, es decir su misión, sus objetivos, los servicios que presta, la forma de financiarse, etc. y

- Que la causa principal de esto es o bien que no se tiene una buena política de

Comunicación, o que ésta es ineficaz y habría que optimizarla

Las consecuencias de esta deficiencia comunicativa también vienen expresadas en los resultados de los estudios de campo efectuados:

- Imposibilidad de transmitir un proyecto propio identificable, a partir del cual se abran para la ENL nuevos campos de actividad relacionados con aquel.

- $\quad$ Falta de visibilidad social que origina la inexistencia de una imagen corporativa concreta.

- $\quad$ Carencia de liderazgo social público y de posicionamiento frente a sus competidores y clientes.

Ante estas circunstancias, parecería lógico que una ONG se planteara una política de Comunicación enfocada a sus públicos internos y externos, derivada de las respuestas a las siguientes cuestiones lógicas:

- ¿Qué debe comunicar la ENL?

- ¿ ¿A quién se debe dirigir la Comunicación prioritariamente?

- ¿ ¿De qué forma se debe efectuar esta Comunicación?

\section{Conclusiones}

Es bastante escaso las fuentes investigativas, académicas y productivas en torno a un tipo de marketing aplicable en las organizaciones del tercer sector, cuyos fines son orientados a lo social. Sin embargo, se permite definirlo como una herramienta de apoyo para que las entidades sociales 
alcancen su finalidad de transformar la sociedad en su conjunto y de proporcionarle bienestar y calidad social. La concentración del marketing se da en 2 frentes de trabajo, los grupos de apoyo y la población vulnerable.

Un tipo de marketing adecuado a las especificidades del tercer sector y a las actividades de las organizaciones inmersas en él comienza a tomar fuerza en el contexto latinoamericano. Una modalidad que apoye no solo la labor de fundadores y directivos en su propósito de mejorar la calidad de vida de la población en situaciones de vulnerabilidad, sino también la tarea de los fundraisers de búsqueda y canalización de recursos monetarios y materiales para implementar de forma focalizada y oportuna programas sociales.

Las variables controlables que, integradas y coordinadas, aportan a la satisfacción de necesidades de la población vulnerable y al bienestar de la sociedad en general, que aplicadas de forma integrada y coordinada, además de informar y atraer grupos de apoyo, influyen en la actitud de otros stakeholders igualmente importantes.

El traslado, las interpolaciones y las igualdades entre marketing empresarial y marketing de organizaciones sociales, por lo general desencadenan resultados desafortunados, quienes han tenido que padecer sus desatinos son aquellos estudiantes de administración de empresas que han realizado sus prácticas y pasantías en organizaciones sociales (centros de beneficencia, fundaciones, hogares infantiles, centros de apoyo, etc.).

El asunto central de las organizaciones sociales es el financiamiento, ya que de este depende la ejecución de sus programas, entonces, son necesarias las investigaciones tendientes a la identificación de perfiles de donantes individuales y empresariales, a la ubicación de transferencias gubernamentales en sus 3 niveles (nacional, provincial y municipal) y a la compilación de directorios de agencias y fondos internacionales con recursos para poblaciones vulnerables que demandan ayuda urgente. Los mapas de vulnerabilidad (desplazamiento, desmovilización, abandono, maltrato, etc.) presentados como instrumentos para la focalización de programas sociales constituyen otra posibilidad para los investigadores interesados. 


\section{Referencias bibliográficas}

BARQUERA, S., RIVERA, J. Y GASCA, A. (2001). Políticas y programas de alimentación y nutrición en México. Salud Pública de México, 43, 464-477.

BARRANCO, J. (2018). Marketing Social y Tercer Sector. Tendencias 21. España. Disponible en: https://www.tendencias21.net/marketing/Marketing-Social-y-Tercer-Sector_a20.html

BARRANCO, J. (2018). Marketing para el Tercer Sector. Tendencias 21. España. Disponible en: https://www.tendencias21.net/marketing/Marketing-para-el-Tercer-Sector-2_a117.html

Kotler, P. y Roberto, E. (1992). Marketing social. Madrid: Ediciones Díaz de Santos.

Kotler, P. y Levy, S. (1969). Broadening the Concept of Marketing. Journal of Marketing, 33, 1015.

Latorre, X. (2001). El marketing aplicado a las ONGD: coherencias e incoherencias en relación con la educación para el desarrollo. Comunicar, 16, 103-114.

Liao, M, Foreman, S. y Sargeant, A. (2001). Market versus societal orientation in the nonprofit context. International Journal of Nonprofit and Voluntary Sector Marketing, 6, 254-268.

Lorca, P. (2004). La creación de valor en la empresa y los stakeholders. Finanzas y Contabilidad, $51,49-54$.

Martín, J. y Martínez, J. (2004). La misión y los fondos de las instituciones no lucrativas.

Business Review, 103, 78-85

Richer, M. y López, N. (2004). Un enfoque socioeconómico de las organizaciones del tercer Sector. Espacio Abierto, 13, 587-606.

TOCA, C. (2011). Las organizaciones de la sociedad civil. Retos y posibilidades. Saarbrücken: Editorial Académica Española.

TOCA, C. Y CARRILLO, J. (2011). Donating and Volunteering as Social Capital in Bogotá. Bulletin of Latin American Research BLAR, 32, 133-148. 
TOCA, C., (2013). El marketing al servicio de las organizaciones del tercer sector. Revista ScienceDirect, Volumen 29, págs. 386-395. Disponible en: https://www.sciencedirect.com/science/article/pii/S0123592314000758

VEGA, P. (1997). El panel de expertos. El programa de monitoreo de la política social. Dos experiencias de evaluación de programas públicos. Última Década, 8, 1-13.

Wong, L. y Tang, J. (2006/2007). Dilemmas Confronting Social Entrepreneurs: Care Homes for Elderly People in Chinese Cities. Pacific Affairs, 79, 623-640. 